\title{
Histological and Immunohistological Findings Using Anti-Cortisol Antibody in Atopic Dermatitis with Topical Steroid Addiction
}

Mototsugu Fukaya

To view enhanced content go to www.dermtherapy-open.com Received: December 6, 2015 / Published online: February 2, 2016

(C) The Author(s) 2016. This article is published with open access at Springerlink.com

\section{ABSTRACT}

Introduction: Though topical steroid addiction (TSA) in patients with atopic dermatitis (AD) has been recently discussed as a clinical problem, there are very few studies about its mechanism. The purpose of this study was to elucidate histological and immunohistological characteristics of TSA using anti-cortisol antibody.

Methods: Skin biopsy specimen from eight patients with $\mathrm{AD}$ was stained by anti-cortisol antibody (Biorbyt, orb79379). Subjects consisted of a child patient with a short history of topical corticosteroids (TCS) application, an adult patient with a long history of TCS application, and six adult patients who have experienced topical steroid withdrawal (TSW) and the rebound phenomenon.

Electronic supplementary material The online version of this article (doi:10.1007/s13555-016-0096-7) contains supplementary material, which is available to authorized users.

M. Fukaya $(\bowtie)$

Tsurumai Kouen Clinic, Nagoya, Japan e-mail: moto@earth.ocn.ne.jp
Results: The staining in the epidermis by anti-cortisol antibody presented patchy defects in the child patient, the patient with a long history of TCS application, and two patients at the rebound period. Parakeratosis with poor formation of corneal layer was obvious in the child patient, the patient with a long history of TCS application, two patients recovered from TSA, and two patients at the rebound period.

Conclusion: Prolonged application of TCS might suppress the cortisol production of keratinocytes which is poorly developed at the early ages before childhood and completed naturally as to growth. Rebound phenomenon after TSW can occur due to the relative insufficiency of cortisol in the epidermis and the immature corneal layer formation.

Keywords: Atopic dermatitis; Cortisol; Topical steroid addiction

\section{INTRODUCTION}

Skin has recently been shown to be a cortisol-producing organ and the cortisol controls the skin itself by regulating differentiation process from basal cells to 
corneal cells $[1,2]$. The production of cortisol by keratinocytes is affected by the humidity in the environment [3]. Topical corticosteroids (TCS) may have some influence on the production of cortisol in the skin and its influence can be associated with some side effects of TCS.

There have been many reported side effects with TCS; topical corticosteroid addiction (TSA) $[4,5]$ which is discussed mainly in atopic dermatitis (AD) $[6,7]$ is one such side effect. As the difference between original $\mathrm{AD}$ and TSA is little in the skin manifestation, the conception of TSA has not been widely recognized even among dermatologists. The diagnosis of TSA has been done by the clinical course including ineffectiveness of TCS after prolonged use and severe rebound phenomenon after withdrawal which subsides in months or years without any treatment [7]. The author built a hypothesis that TSA is caused by the suppressed self-production of cortisol in the keratinocytes and conducted an immunohistological study using anti-cortisol antibody in patients with AD.

\section{METHODS}

Eight patients with $\mathrm{AD}$ and one healthy individual participated in the study. Skin biopsy was performed from the flexor side of the forearm near elbow. The specimen was fixed by formaldehyde and sent to the laboratory (Morphotechnology, Co., Ltd, Sapporo Japan). The paraffin-embedded specimens were treated by $\mathrm{pH} 6$ citrate buffer solution for $20 \mathrm{~min}$ at $95-98^{\circ} \mathrm{C}$ for the purpose of antigen retrieval. The monoclonal antibody against human cortisol from Byorbyt Co., Ltd (catalogue number : orb79379) was used as the primary antibody. The dilution ratio was 1:200 and reacting time was overnight.
All procedures followed were in accordance with the ethical standards of the responsible committee on human experimentation (institutional and national) and with the Helsinki Declaration of 1964, as revised in 2013. Informed consent was obtained from all patients for being included in the study.

\section{RESULTS}

Cortisol in the epidermis was stained homogeneously in healthy individuals by the preliminary study and in all three patients recovered from TSA (Fig. 1, case 3, 4, and 5). The histological difference among patients with active and inactive eczema in the three cases was in the existence of spongiosis and parakeratosis.

A patient with insistent lesion on the face long after TSW also presented parakeratosis and immature corneal layer while the patient showed normal keratosis in the armpit (Fig. 2, case 4$)$.

A child patient with a short history of TCS application (Fig. 3, case 1), an adult patient with a long history of TCS application (Fig. 3, case 2) and two patients with rebound phenomenon after TSW (Fig. 4, case 6 and 7) whose skin manifestations were obviously different from those of typical AD showed patchy defect of cortisol staining in the epidermis. One of the two patients with rebound was re-examined after improvement in 1 year and his epidermis showed homogeneous pattern in cortisol staining (Fig. 2, case 7).

A patient with rebound phenomenon after TSW whose skin lesion expanded geographically and disappeared as its development showed homogeneous pattern in cortisol staining while parakeratosis with 

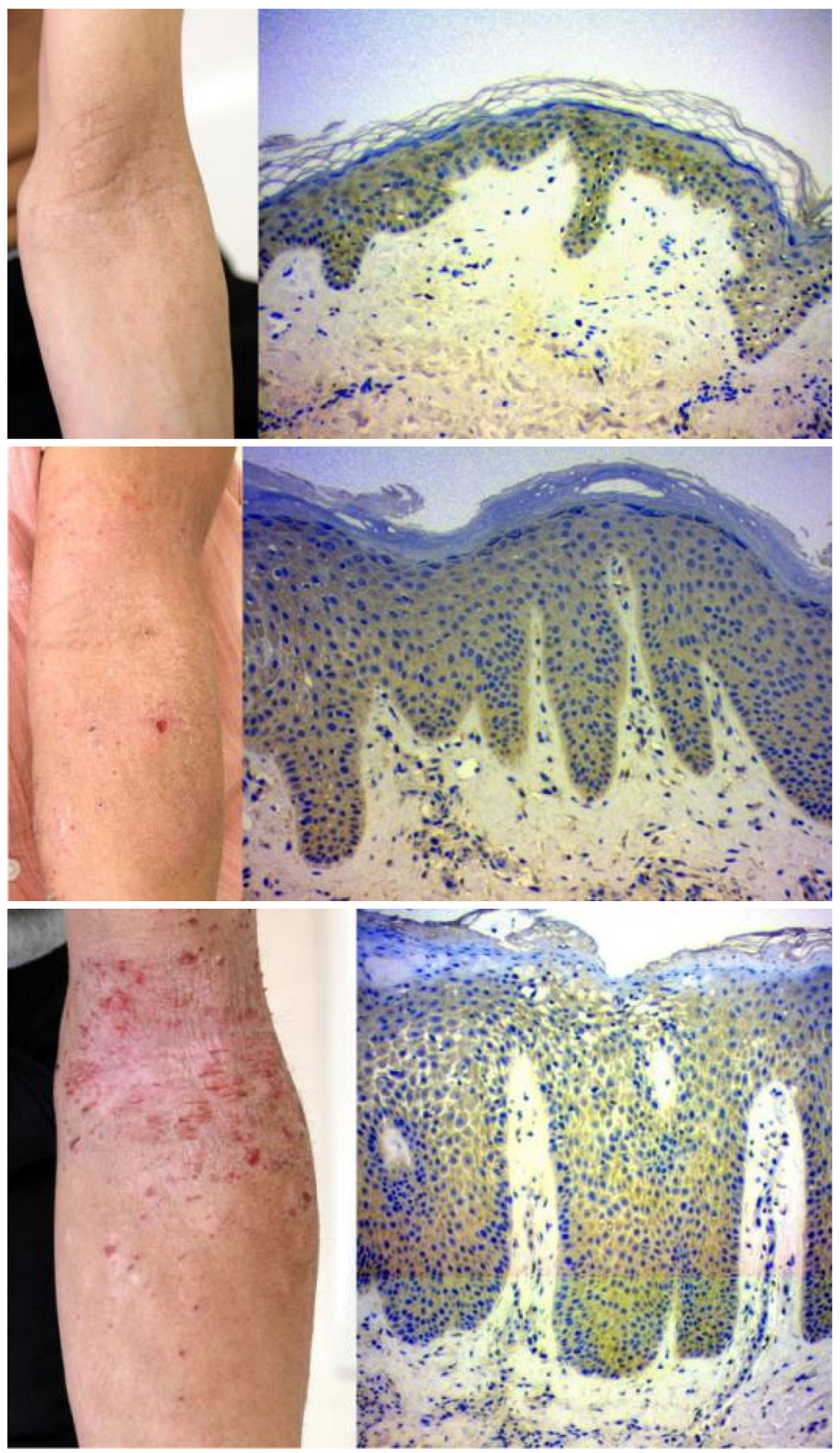

Fig. 1 Three adult patients recovered from TSA. Case 3 (upper): Almost normal clinical and histological appearance. Case 4 (middle): Slight chronic eczema with histologically thickened epidermis. Case 5 (lower): Acute exacerbated eczema with spongiosis and parakeratosis. Note that the eczema is located on the armpit that is the predilection site of atopic dermatitis without TSA. All cases presented the homogeneous pattern of cortisol staining in the epidermis. TSA Topical steroid addiction 


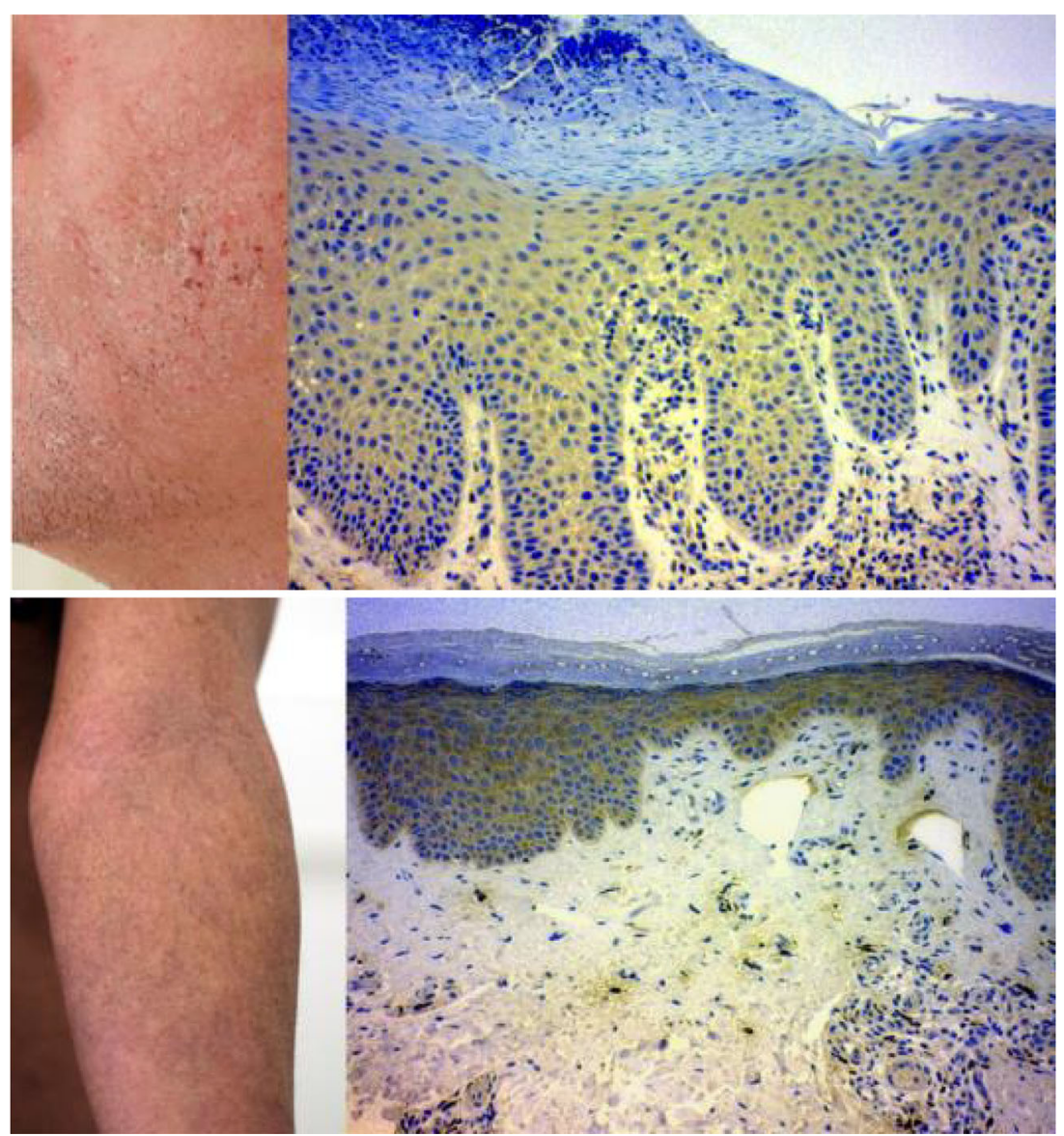

Fig. 2 Supplementation for case 4 (upper) and case 7 (lower). The upper is the cheek in which insistent eczema continued in case 4. The cortisol staining was homogeneous, but the corneal layer remained immature. The eczema was mild in the armpit in which corneal layer

immature corneal layer was prominent (Fig. 4, case 8$)$.

\section{DISCUSSION}

There has been little reported about the histological or immunohistological characteristics of TSA in AD. Sheu et al. [8, 9] reported that parakeratosis and insufficient corneal layer formation were characteristic in patients with TSA by examining the inconsistent erythema on the face. The finding coincides with our cases (case 4 cheek, case 5, 6, 7, and 8). was mature (Fig. 1, middle). The lower is the improved appearance after 1 year without resuming topical corticosteroid in case 7. The cortisol staining in the epidermis became homogeneous suggesting the recovery of self-production of cortisol in keratinocytes

It is well known that cortisol production from the adrenal gland is suppressed by long-term use of systemic steroids [10]. As far as the skin is also the organ which produces cortisol, its function can be influenced by the long-term application of TCS. The fact that epidermis becomes thin and atrophic after prolonged use of TCS and temporarily thickens after withdrawal [11] can be explained by that mechanism.

The epidermis is a barrier against external stimuli or allergens. Cortisol production by keratinocytes might work to regulate or 


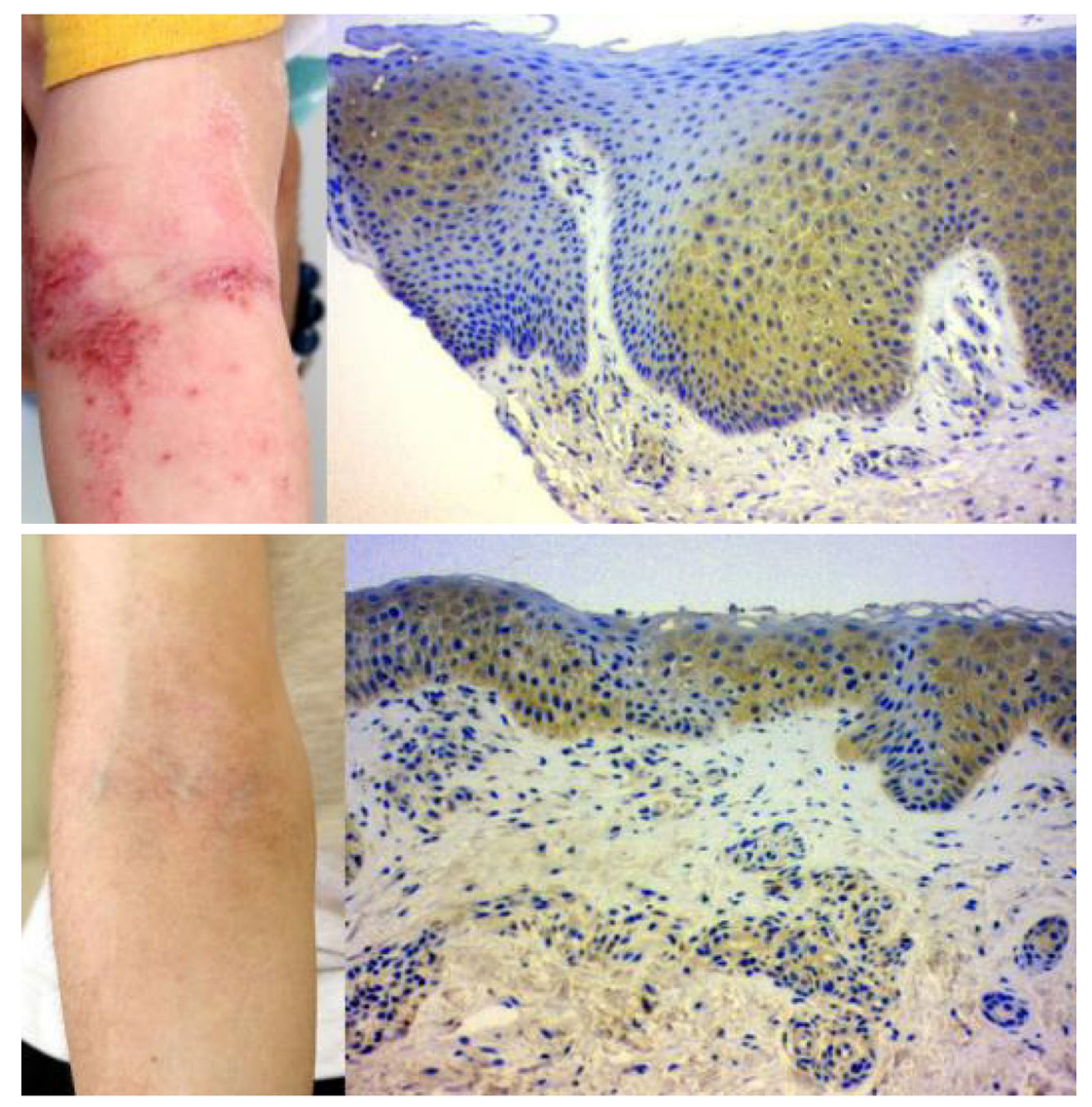

Fig. 3 The child patient with a short history of TCS application (case 1, upper) and the adult patient with a long history of TCS application (case 2, lower). Both showed patchy defect of cortisol staining and prominent parakeratosis with immature corneal layer. Case 2 was apparently well controlled by TCS with the epidermis of normal thickness and few inflamed cells in the dermis. TCS Topical corticosteroid moderate the friction between the outer environment and inner immune system by suppressing excessive inflammation or immune reaction. However, prolonged or excessive use of TCS induces skin atrophy which can make barrier function weak. Moreover, the decreased self-production of cortisol by the keratinocytes can cause hypersensitivity. The author considers it is one of the mechanisms of TSA or rebound phenomenon after TSW.

In this study, a child patient with short-term history of TCS application presented patchy defect of cortisol production in the epidermis as with the adult patients with TSA, while the pattern was homogeneous in a healthy adult individual. The author suspects that the production of cortisol in keratinocytes is immature at early ages before childhood and that is the reason why eczema is developed at such ages and heals naturally with growth. Keratinocytes will become mature and begin to produce cortisol homogeneously as to growth.

Parakeratosis or immature corneal layer formation which remains incontinently long after TSW as in case 4 (cheek) might be associated with another mechanism of TSA 

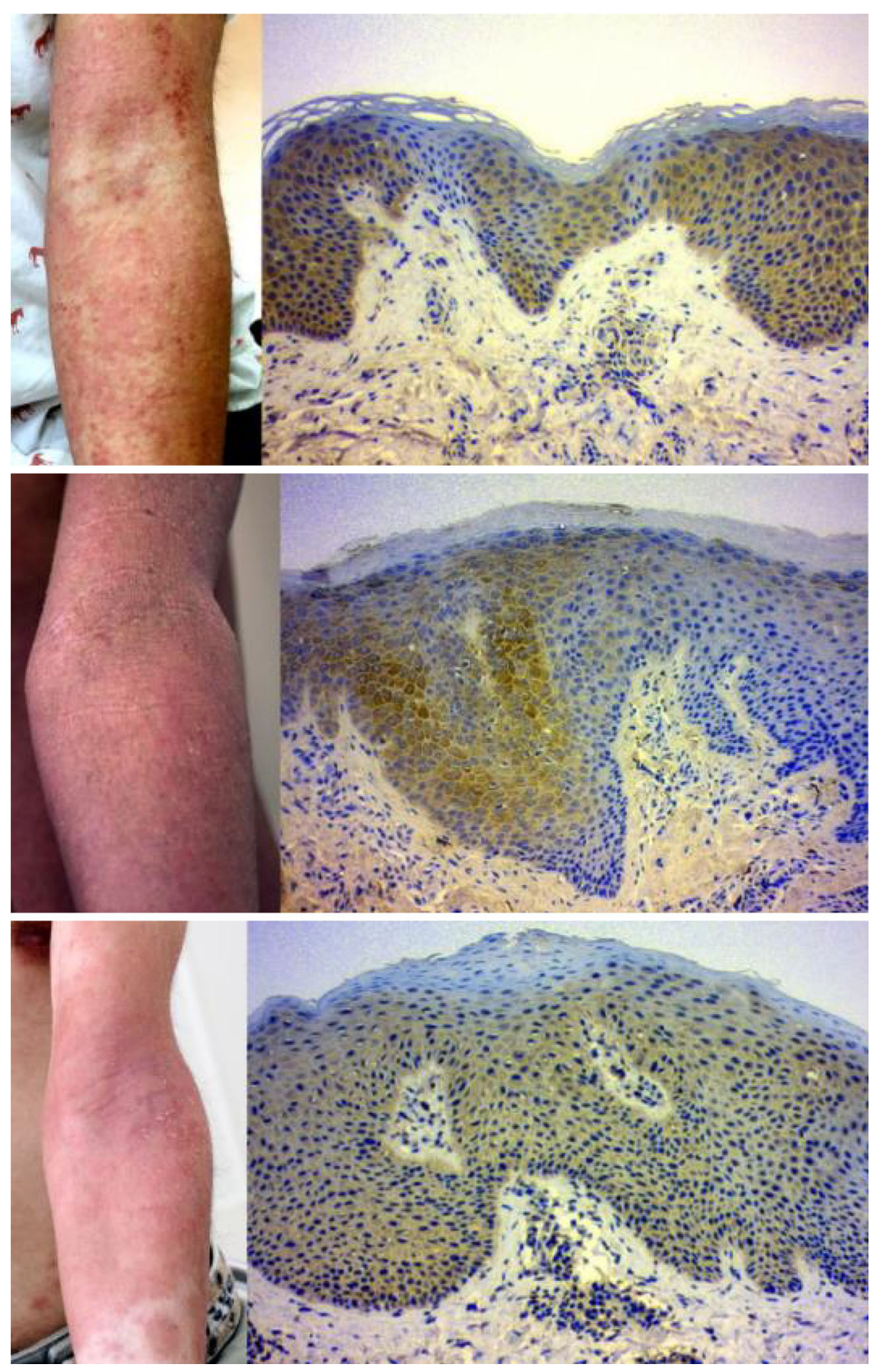

Fig. 4 Three adult patients during the rebound phenomenon after TSW. Case 6 (upper): Diffuse papulo-erythematous lesion that is atypical as atopic dermatitis, but typical as topical steroid addiction. Case 7 (middle): Erythroderma. Case 8 (lower): Demarcated

because the cortisol staining in the epidermis was homogeneous. However, the possibility that the cortisol production in the epidermis is geographic erythema that is one typical pattern developing after TSW. Case 6 and 7 showed patchy defect of cortisol staining in the epidermis. Case 8 presented prominent parakeratosis with homogeneous pattern of cortisol staining. TSW Topical steroid withdrawal

decreasing in such cases still remains because the immunohistological study was not quantitative. 
The author admits that the population of the cases is too small to prove the above hypothesis. There is a possibility that further studies put a different interpretation. So the study should be regarded only as a preliminary one.

\section{CONCLUSIONS}

Prolonged application of TCS might suppress the cortisol production of keratinocytes which is poorly developed at the early ages before childhood and completed naturally as to growth. Rebound phenomenon after TSW can occur due to the relative insufficiency of cortisol in the epidermis and the immature corneal layer formation.

\section{ACKNOWLEDGMENTS}

No funding or sponsorship was received for this study or publication of this article. The named author meets the International Committee of Medical Journal Editors (ICMJE) criteria for authorship for this manuscript, takes responsibility for the integrity of the work as a whole, and has given final approval for the version to be published.

Disclosures. Mototsugu Fukaya has nothing to disclose.

Compliance with ethics guidelines. All procedures followed were in accordance with the ethical standards of the responsible committee on human experimentation (institutional and national) and with the Helsinki Declaration of 1964, as revised in 2013. Informed consent was obtained from all patients for being included in the study.
Open Access. This article is distributed under the terms of the Creative Commons AttributionNonCommercial 4.0 International License (http://creativecommons.org/licenses/by-nc/4. $0 /$ ), which permits any noncommercial use, distribution, and reproduction in any medium, provided you give appropriate credit to the original author(s) and the source, provide a link to the Creative Commons license, and indicate if changes were made.

\section{REFERENCES}

1. Cirillo N, Prime SS. Keratinocytes synthesize and activate cortisol. J Cell Biochem. 2011;112(6):1499-505.

2. Slominski A, Zbytek B, Nikolakis G, Manna PR, Skobowiat C, Zmijewski M, Li W, Janjetovic Z, Postlethwaite A, Zouboulis CC, Tuckey R, et al. Steroidogenesis in the skin: implications for local immune functions. J Steroid Biochem Mol Biol. 2013;137:107-23.

3. Takei K, Denda S, Kumamoto J, Denda M. Low environmental humidity induces synthesis and release of cortisol in an epidermal organotypic culture system. Exp Dermatol. 2013;22(10):662-4.

4. Kligman AM, Frosch PJ. Steroid addiction. Int J Dermatol. 1979;18(1):23-31.

5. Rapaport MJ, Rapaport V. Prolonged erythema after facial laser resurfacing or phenol peel secondary to corticosteroid addiction. Dermatol Surg. 1999;25(10):781-4 (discussion 785).

6. Hajar T, Leshem YA, Hanifin JM, the National Eczema Association Task Force, et al. A systematic review of topical corticosteroid withdrawal ("steroid addiction") in patients with atopic dermatitis and other dermatoses. J Am Acad Dermatol. 2015;72(3):541-9.

7. Fukaya M, Sato K, Sato M, et al. Topical steroid addiction in atopic dermatitis. Drug Healthc Patient Saf. 2014;14(6):131-8.

8. Sheu HM, Tai CL, Kuo KW, Yu HS, Chai CY. Modulation of epidermal terminal differentiation in patients after long-term topical corticosteroids. J Dermatol. 1991;18(8):454-64. 
9. Sheu HM, Chang CH. Alterations in water content of the stratum corneum following long-term topical corticosteroids. J Formos Med Assoc. 1991;90(7):664-9.

10. McIntosh HW, Holmes CB. Some evidence suggesting the suppression of adrenocortical function by cortisone. Lancet. 1951;2(6693):1061-4.
11. Zheng PS, Lavker RM, Lehmann P, Kligman AM. Morphologic investigations on the rebound phenomenon after corticosteroid-induced atrophy in human skin. J Invest Dermatol. 1984;82(4):345-52. 